\title{
REMOCIÓN DE IONES COBRE CON SORBENTES ORGÁNICOS
}

\author{
P. Tapia ${ }^{1}$, O. Pavez ${ }^{1,2}$, M. Santander ${ }^{1}$, B. Sepúlveda ${ }^{2 *}$ \\ ${ }^{1}$ Departamento de Ingeniería en Metalurgia, Universidad de Atacama ${ }^{2}$ Centro Regional de Investigación \\ y Desarrollo Sustentable de Atacama - CRIDESAT Av. Copayapu 485, Copiapó, Chile \\ patricia.tapia@uda.cl - osvaldo.pavez@uda.cl - mario.santanderquda.cl - *bernardo.sepulveda@uda.cl
}

Submetido 11/12/2017 - Aceito 28/12/2017

DOI: $10.15628 /$ holos.2017.6661

\section{RESUMEN}

Se estudió la remoción de iones de cobre desde soluciones acuosas artificiales empleando diferentes materiales sorbentes orgánicos: cáscaras de nuezalmendra, cáscaras de avellana, cáscaras de maní, aserrín, cuescos de durazno, algas. Las pruebas de sorción se realizaron a escala batch estudiándose la cinética de sorción de los iones cobre y los efectos en la remoción de cobre de la concentración del sorbente, la concentración inicial de cobre y la fuerza iónica de la solución. Los resultados obtenidos muestran que todos los sorbentes producen remociones parciales de cobre, sin embargo al comparar la efectividad de ellos, las cáscaras de nuezalmendra, el aserrín y las cáscaras de maní fueron más efectivos en la remoción de cobre, mientras que los cuescos de durazno resultaron menos adecuados. Los datos de equilibrio de adsorción de cobre en función del tiempo de contacto con el sorbente, se analizaron mediante las ecuaciones de isotermas de adsorción de Freundlich, Langmuir y BET, obteniéndose con la ecuación de Freundlich coeficientes de correlación $\left(R^{2}\right)$ del orden de 0,99 mostrando que este modelo representaba en forma más adecuada el proceso de sorción de los iones de cobre en los sorbentes utilizados. La fuerza iónica de la solución fue estudiada a través de la aplicación de diferentes concentraciones de $\mathrm{NaCl}$ encontrándose que la remoción de cobre disminuía al aumentar la fuerza iónica.

Palabras claves: Sorbentes orgánicos, remoción, iones cobre, isotermas de adsorción.

\section{REMOVAL OF COPPER IONS WITH ORGANIC SORBENTS}

\begin{abstract}
Copper ions removal was studied from aqueous solutions using different artificial organic sorbent materials: walnut-almond shells, hazelnut shells, peanut hulls, sawdust, peach pits and algae. Sorption tests scale batch were conducted to study the kinetics of sorption of ions copper and the effects of the sorbent concentration, initial concentration of copper and the ionic strength of the solution. The results showed that the sorbents produce a partial removal of copper, however to compare the effectiveness of them, nutshells, almond, sawdust and peanut shells
\end{abstract}

were more effective in the removal of copper, while the peach pits were less suitable. The equilibrium data of copper adsorption were analyzed using Freundlich, Langmuir and BET equations, obtained with the Freundlich equation correlation coefficients $\left(R^{2}\right)$ of 0.99 , showing that this model was more adequate to represent the sorption of copper ions in the sorbents. The ionic strength of the solution was studied through the application of different $\mathrm{NaCl}$ concentrations, obtaining that the copper removal decreased with increasing ionic strength.

Keywords: Organic sorbents, removal, copper ions, adsorption isotherms 


\section{INTRODUCCIÓN}

Los recursos hídricos son muy importantes en los procesos productivos, sin embargo, una vez utilizados son descargados al medio ambiente con sus propiedades físicas y químicas modificadas. Debido a esta situación, los organismos de control ambiental han establecido normas de descarga cada vez más estrictas. Para cumplir con estas normas más exigentes, en actividades industriales se ha hecho necesario tratar los residuos líquidos antes de descargarlos al medio ambiente, incluso cuando éstos son recirculados dentro del proceso; debido a que la carga contaminante puede generar alteraciones en las operaciones de los procesos productivos. Entre los principales contaminantes asociados a los residuos líquidos de las distintas actividades industriales se encuentran los metales pesados.

La toxicidad de los metales pesados y su efecto en el ecosistema han generado en los últimos años un incremento considerable de estudios relacionados con su remoción desde soluciones acuosas. Normalmente, son utilizados diferentes procesos para la descontaminación de soluciones que contienen metales pesados, entre ellos se pueden destacar: precipitación/sedimentación/filtración, flotación, intercambio iónico, micro/nano/ultrafiltración, osmosis reversa. Sin embargo, la aplicación de estos procesos muchas veces resulta restrictiva por razones económicas y/o por no ser eficaz para el tratamiento de soluciones muy diluidas. Por ello, se torna interesante investigar y desarrollar materiales sorbentes simples, de bajo costo, que puedan ser utilizados en el tratamiento de aguas residuales para la remoción de metales pesados.

En este contexto, la factibilidad comercial de la remoción de metales pesados mediante procesos de biosorción se torna atractiva. La utilización de elementos de origen biológico se presenta como un proceso de tratamiento rentable que puede hacer descender el nivel de materiales tóxicos por debajo de los niveles máximos permitido, de modo de disminuir la potencial contaminación de los recursos hídricos disponibles y posibilitar que las aguas recuperadas puedan albergar vida silvestre o ser utilizadas para la agricultura u otros fines.

En este sentido, el presente trabajo tiene como objetivo estudiar la efectividad de diversos materiales sorbentes orgánicos en la remoción de iones cobre desde soluciones acuosas artificiales. Los materiales que se utilizan en este estudio, caracterizados por ser de muy bajo costo, son los siguientes: cáscaras de maní, cáscaras de avellana, cascaras de nuez-almendra, aserrín, cuescos de durazno y algas. Se estima que el desarrollo de este estudio es un aporte al conocimiento e investigación del uso de materiales sorbentes simples y de bajo costo para la eliminación de metales pesados.

\section{REVISIÓN BIBLIOGRÁFICA}

Frente a las tecnologías convencionales utilizadas en la eliminación de metales pesados desde soluciones, las investigaciones se han orientado en la búsqueda de procesos y materiales alternativos de bajo costo. El uso de sistemas biológicos para la remoción de metales pesados en soluciones diluidas, presenta un gran potencial para la obtención de mejores resultados y a un menor costo, lo cual ha llevado al desarrollo de estas tecnologías. Dentro de los tratamientos biológicos, la tecnología que utiliza procesos de biosorción es operacional y económicamente competitiva, por lo cual, la biosorción aplicando materiales orgánicos, es considerada una alternativa tecnológica prometedora para el tratamiento de aguas residuales que contienen metales pesados. 
En la literatura se reportan diversos materiales orgánicos que han mostrado efectividad en la remoción de iones cobre. Kondo et al. (1996) estudiaron la adsorción de iones metálicos en quitosan químicamente modificado por D-galactosa, ellos indican que el orden de adsorción de los iones metálicos en este material fue $\mathrm{Cu}>\mathrm{Ni}>\mathrm{Co}$. Singh et al. (2013) utilizaron diferentes residuos agrícolas en la remoción de $\mathrm{Ni}^{2+}, \mathrm{Cu}^{2+}$ y $\mathrm{Zn}^{2+}$ desde soluciones acuosas, encontrando que las mayores capacidades alcanzadas fueron $92,52,94,12$ y $96,75 \mathrm{mg} / \mathrm{g}$, respectivamente. La potencialidad de biomasas de residuos agrícolas naturales en la remoción de iones $\mathrm{Cu}(\mathrm{II})$ desde soluciones acuosas también fue reportada recientemente por Singha y Das (2013). La utilización de residuos de manzana en la remoción de cobre desde solución acuosa fue estudiada por Lee y Yang (1997), quienes evaluaron las capacidades de intercambio bio-catión de estos residuos analizando el efecto del pH, fuerza iónica, co-ión, ligandos, concentración inicial del metal y tamaño de las partículas de los residuos de manzana. Residuos agrícolas de bajo costo han sido utilizados para la biosorción de cobre (Bandela, Bagrekar, Jogdand y Kaushik, 2016; Mohammed, Azeez y Lutffe, 2015) y para la remoción de diferentes metales pesados (incluyendo cobre) desde aguas residuales (Tripathi y Ranjan, 2015). Cáscaras de maní fueron utilizadas en la eliminación de $\mathrm{Cu}(\mathrm{II})$ por Zhu et al. (2009), quienes encontraron que la remoción era dependiente del pH alcanzándose un máximo en pH 5,5, además informaron que el proceso de biosorción seguía una cinética de pseudo segundo orden y el equilibrio se alcanzaba a las dos horas. También Khan et al. (2015) utilizaron cáscaras de maní en la remoción de cobre y níquel obteniendo buenos resultados. Cáscaras de nueces fueron utilizadas en la biosorption de iones cobre, variando $\mathrm{pH}$, tiempo de contacto y dosis del adsorbente, alcanzándose una máxima remoción de cobre del 79,5\% (Kamar y Nechifor, 2015). Alguacil y Merino (1998) haciendo una revisión sobre biotratamientos de contaminantes de origen orgánico reportaron microorganismos para la biosorción de metales, destacando la remoción de cobre con bacterias (Bacillus sp, Streptomyces noursei), hongos (Penicillum spinulosum, Penicillum crhysogenum, Aspergillus niger Penicillum), levadura (Sacchanomyces Cerevisiae) y algas (Vaucheria). Estudios con Rhizopus Arrhizuz para remover simultáneamente $\mathrm{Cr}(\mathrm{IV})$ y $\mathrm{Cu}(\mathrm{II})$ fueron reportados por Sag et al. (1999). Saeed et al. (2005) evaluaron madera de la papaya como un nuevo biosorbente de metales pesados, ellos encontraron que la sorción de iones era más eficiente en $\mathrm{pH} 5$, alcanzándose remociones de $99,7 \%, 94,7 \%$ y $66,8 \%$ para cobre, cadmio y zinc, respectivamente. Estudios sobre el efecto de un tratamiento oxidante a un substrato de lignocelulosa en la sorción de $\mathrm{Cu}$ y $\mathrm{Zn}$, empleando como agentes oxidantes permanganato de potasio $\left(\mathrm{KMnO}_{4}\right)$ o periodato de sodio $\left(\mathrm{NaIO}_{4}\right)$, fueron realizados por Jolly et al. (2006), quienes concluyeron que la lignocelulosa oxidada es eficiente y se presenta como un biomaterial promisorio para la descontaminación de las aguas residuales. Deng et al. (2006) investigaron la biosorción de $\mathrm{Cu}^{2+}$ y $\mathrm{Pb}^{2+}$ mediante Cladophora fascicularis en función del pH inicial, concentración inicial de los metales pesados, temperatura y otros iones coexistentes, mostrando que la Cladophora fascicularis era un efectivo y económico material biosorbente para la remoción y recuperación de iones de metales pesados desde aguas residuales. Bal et al. (2006) emplearon biomasa de pleurotus mutilus, chitin y chitosan para la remoción de iones $\mathrm{Cu}$ (II) desde soluciones acuosas, ellos indicaron que la biomasa fue tan eficiente como el chitin y permitió un rendimiento máximo en pH en torno a 6. Radu et al. (2006) utilizaron residuos biológicos de diferentes procesos (tecnología farmaceutica, industria alimenticia, entre otros), como material biosorbente para mejorar la calidad de las aguas residuales industriales, ellos reportaron resultados alcanzados con estos residuos biológicos en la remoción de iones cobre y cobalto desde soluciones acuosas. Luna et al. (2007) realizaron investigación básica para conocer la remoción de iones cobre desde soluciones acuosas empleando Sargassum filipéndula en condiciones batch, estudiando parámetros experimentales 
tales como pH, tiempo de sorción, condiciones de equilibrio y concentración inicial de los iones cobre. Estudios sobre la biosorción de iones cobre mediante biomasas de algas marinas han sido reportados en la literatura (Sheng, Wee, Ting y Chen, 2008; Vilar, Botelho, Pinheiro, Domingos y Boaventura, 2009; Fagundes-Klen, Veit, Borba, Bergamasco, Vaz y da Silva, 2010; Lahari, King y Prasad, 2011; Tapia, Santander, Pavez, Guzmán y Romero, 2011; Estefandian, Javadian, Parvini, Khoshandam y Katal, 2013; Kushwah y Srivastav, 2015). Tratamientos biológicos usando lechos microbianos conteniendo un lecho flotante de algas verde-azules que permitían el desarrollo de Biomat (nombre comercial del lecho), han sido aplicados en la eliminación de cobre (Berder y Phillips, 1996). Recientemente se ha reportado el uso de liquen en la remoción de iones cobre y níquel (Candan, Tay, Avan y Tay, 2017).

\section{METODOLOGIA}

\subsection{Sorbentes orgánicos}

En este estudio se utilizaron como sorbentes orgánicos, cáscaras de maní, cáscaras de nuezalmendra, algas, aserrín, cáscaras de avellana y cuescos de durazno, los cuales no tuvieron un pretratamiento para su utilización. En la Tabla I, Tabla II y Tabla III se presentan las características físicas y la composición química de los sorbentes, respectivamente.

Tabla I: Características físicas de los sorbentes orgánicos.

\begin{tabular}{c|c|c}
\hline Sorbente & Tamaño $[\mu \mathrm{m}]$ & Densidad aparente $\left[\mathrm{kg} / \mathrm{m}^{3}\right]$ \\
\hline Cáscara de maní & $100 \%<9423$ & 132 \\
Nuez-Almendra & $100 \%<1651$ & 573 \\
Algas & - & 64 \\
Aserrín & $100 \%<6680$ & 93 \\
Avellana & $100 \%<3327$ & 285 \\
Cuesco Durazno & $100 \%<4699$ & 515 \\
\hline
\end{tabular}

Tabla II: Composición química de las algas.

\begin{tabular}{c|c|c|c|c|c}
\hline \multicolumn{4}{c|}{ Caracterización iónica[mg/L] } & \multicolumn{2}{c}{ PIGMENTOS [MG/ML] } \\
\hline NA & K & MG & CA & CLOROFILA TOTAL & CAROTENOIDES TOTAL \\
664 & 724 & 300 & 339 & 3,621 & 0,398 \\
\hline
\end{tabular}

Tabla III: Composición química de los sorbentes orgánicos.

\begin{tabular}{c|c|c|c}
\hline \multirow{2}{*}{ Sorbente } & \multicolumn{2}{|c}{ Compuesto [ABS/g sorbente] } \\
\cline { 2 - 4 } & Lignina $\lambda=300 \mathrm{~nm}$ & Tanino $\lambda=540 \mathrm{~nm}$ & Celulosa $\lambda=500 \mathrm{~nm}$ \\
\hline Cuesco de durazno & 0,1575 & 0,0145 & 0,5250 \\
Avellana & 0,3025 & 0,1208 & 0,3075 \\
Nuez-Almendra & 0,1288 & 0,0403 & 0,3750 \\
Aserrín & 0,3025 & 0,0256 & 0,2250 \\
Cáscara de maní & 0,2650 & 0,0491 & 0,2458 \\
\hline
\end{tabular}

$\lambda$ : Longitud de onda de máxima absorción. 
En las algas, la remoción para caracterizar los iones alcalinos y alcalinotérreos $(\mathrm{Na}, \mathrm{K}, \mathrm{Mg}$,

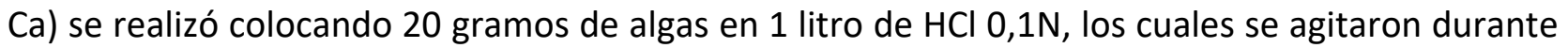
2 horas. Para la determinación de la clorofila se efectuaron previamente extracciones a 2 gramos de sorbente con etanol al 96\%, la mezcla se centrifugó y posteriormente se leyó la absorbancia (ABS) a longitudes de onda de 649 y $665 \mathrm{~nm}$, determinándose el contenido de clorofila. En el caso de la determinación del contenido de proteínas, se confeccionó la curva standard correspondiente y se leyó la absorbancia del extracto en la longitud de onda de $595 \mathrm{~nm}$.

En el resto de los sorbentes utilizados en este estudio, para la determinación de la celulosa se realizó previamente una extracción ácida a cada sorbente con $\mathrm{H}_{2} \mathrm{SO}_{4}$ al $60 \%$, mientras que, en la determinación de la lignina y del tanino se realizaron extracciones con una solución del $\mathrm{HCl}$ al $30 \%$, leyéndose luego la absorbancia en un espectrofotómetro visible en la longitud de onda de máxima absorción del complejo coloreado azul.

En relación a la caracterización granulométrica de los sorbentes, no se hizo un análisis granulométrico de éstos ya que no se requería la distribución granulométrica del material. Por lo tanto, la granulometría del sorbente quedó definida por la abertura del tamiz por el cual se pasó el material, obteniéndose en cada caso sorbente con tamaño $100 \%$ menor a la abertura de tamiz utilizado.

\subsection{Soluciones artificiales de cobre}

Las soluciones artificiales de cobre fueron preparadas con el reactivo $\mathrm{CuSO}_{4} .5 \mathrm{H}_{2} \mathrm{O}$ de $99 \%$ de pureza (Fluka) disuelto en $300 \mathrm{~mL}$ de agua desmineralizada, utilizando un agitador magnético marca Thermoline Modelo Nova. Posteriormente, a través de dilución se prepararon soluciones de cobre para cada prueba, ajustándose el pH de la solución con $\mathrm{H}_{2} \mathrm{SO}_{4}$ y $\mathrm{NaOH}$, ambos de pureza analítica. El volumen total de solución de cobre preparada para cada set de experiencias fue de 20 litros, una vez terminada la preparación de la solución se tomaron muestras para determinar la concentración inicial de cobre. El agua desmineralizada utilizada en la preparación de las soluciones de cobre presentaba las siguientes características: $0,10 \mathrm{mg} / \mathrm{L} \mathrm{Ca} ; 0,02 \mathrm{mg} / \mathrm{L} \mathrm{Mg}$; menos de $2,0 \mathrm{mg} / \mathrm{L} \mathrm{Cl}$; y conductividad eléctrica de $6,77 \mu \mathrm{S} / \mathrm{cm}$.

\subsection{Ensayos de sorción}

Los ensayos de sorción fueron realizados en un sistema batch el cual consistía en un vaso precipitado de $1000 \mathrm{~mL}$ donde se agitaba magnéticamente la solución de cobre conteniendo el sorbente, a 700 RPM. Terminado el tiempo de agitación, la mezcla fue prefiltrada en un tamiz de malla Tyler 10, retirándose los sólidos gruesos. La solución prefiltrada fue nuevamente filtrada en un papel Whatman № 2, que presentaba tamaños de poros de 8 micrones. Posteriormente, a la solución filtrada obtenida se le midió el pH final y se tomó una alícuota de $100 \mathrm{~mL}$ de muestra para determinar la concentración residual de cobre en la solución. La medición del pH se realizó con un pH metro marca HANNA HI8424, mientras que, la determinación de las concentraciones iniciales y finales de cobre se efectuó en un espectrofotómetro de absorción atómica (GBC 908).

En los ensayos de sorción donde estudió el efecto del tiempo de contacto se utilizaron los siguientes datos: concentración inicial del sorbente, $20 \mathrm{~g} / \mathrm{L}$; concentración inicial de cobre, $20 \mathrm{mg} / \mathrm{L} ; \mathrm{pH}, 5,06$. Respecto de los ensayos en los cuales se estudió el efecto de la concentración del sorbente, los datos aplicados fueron los siguientes: concentración inicial de cobre, $20 \mathrm{mg} / \mathrm{L}$; tiempo de contacto, $15 \mathrm{~min}, \mathrm{pH}, 5,01$. Por otra parte, en las pruebas realizadas para el estudio del efecto de la fuerza iónica en la remoción de cobre, se aplicaron los siguientes datos: concentración del sorbente, $20 \mathrm{~g} / \mathrm{L}$; concentración inicial de cobre, $20 \mathrm{mg} / \mathrm{L} ; \mathrm{pH}, 5,2$; tiempo de 
contacto, $15 \mathrm{~min}$. Todos los ensayos de sorción fueron realizados a temperatura entre $15^{\circ} \mathrm{C}$ a $17^{\circ} \mathrm{C}$.

Para la determinación del efecto del tiempo de contacto en la remoción de iones de cobre se realizaron cuatro ensayos para cada sorbente con tiempos de agitación de $5 \mathrm{~min}, 15 \mathrm{~min}, 30$ min y $60 \mathrm{~min}$. En estos ensayos se mantuvo constante la concentración del sorbente, el pH y la concentración inicial de cobre.

El efecto de la concentración del sorbente en la eliminación de iones cobre se estudió a través de tres pruebas aplicadas por cada sorbente, en ellas se utilizaron concentraciones de 10 $\mathrm{g} / \mathrm{L}, 20 \mathrm{~g} / \mathrm{L}$ y $40 \mathrm{~g} / \mathrm{L}$ manteniendo constante el tiempo de contacto, el pH y la concentración inicial de cobre.

La capación de cobre por los diferentes sorbentes fue analizado realizando seis ensayos para cada sorbente variando la concentración inicial de cobre, las concentraciones aplicadas fueron $5 \mathrm{mg} / \mathrm{L}, 10 \mathrm{mg} / \mathrm{L}, 15 \mathrm{mg} / \mathrm{L}, 20 \mathrm{mg} / \mathrm{L}, 25 \mathrm{mg} / \mathrm{L}$ y $30 \mathrm{mg} / \mathrm{L}$. En estos ensayos se mantuvo constante el tiempo de contacto, el pH y la concentración de sorbente.

El efecto de la fuerza iónica de la solución en la remoción de iones cobre fue estudiada para los sorbente alga, aserrín y cascara de maní, utilizando dos concentraciones de $\mathrm{NaCl}$ y manteniendo constante el pH, la concentración inicial de cobre y la concentración del sorbente.

\section{RESULTADOS Y DISCUSIÓN}

\subsection{Efecto del tiempo de contacto en la sorción de iones cobre}

La Figuras 1 y Figura 2 muestran, respectivamente, la concentración residual de cobre en la solución y la remoción de cobre en función del tiempo de contacto, empleando $200 \mathrm{~mL}$ de solución, una concentración inicial de cobre de $20 \mathrm{mg} / \mathrm{L}$, pH 5,06 y concentración de sorbente de $20 \mathrm{~g} / \mathrm{L}$. En la Figura 1 se observa que la concentración final de cobre para todos los tiempos es superior a $6 \mathrm{mg} / \mathrm{L}$, encontrándose que la cáscara de maní, el aserrín y la cáscara de nuezalmendra fueron los sorbentes más efectivos, notándose además que la diferencia observada en los resultados alcanzados con estos sorbentes con respecto a los otros, se hace más notoria a los 30 y 60 minutos de contacto. La Figura 2 muestra que a medida que aumenta el tiempo de contacto se incrementa la remoción de cobre, obteniéndose con las cáscaras de maní los mejores resultados en el rango de tiempo comprendido entre los 5 y 30 minutos, mientras que a los 60 minutos las mayores remociones de cobre se alcanzan con aserrín. De igual forma, en la Figura 2 se observa que en todo el rango de tiempo estudiado los resultados más bajos fueron obtenidos con cuesco de duraznos. Si se considera la composición química de los sorbentes orgánicos que se muestra en la tabla III, se aprecia que el aserrín y la cáscara de maní son los sorbentes que presentan el nivel más bajo de celulosa y el mayor contenido de lignina; mientras que el cuesco de durazno presenta el nivel de celulosa más alto y el menor contenido de lignina. 


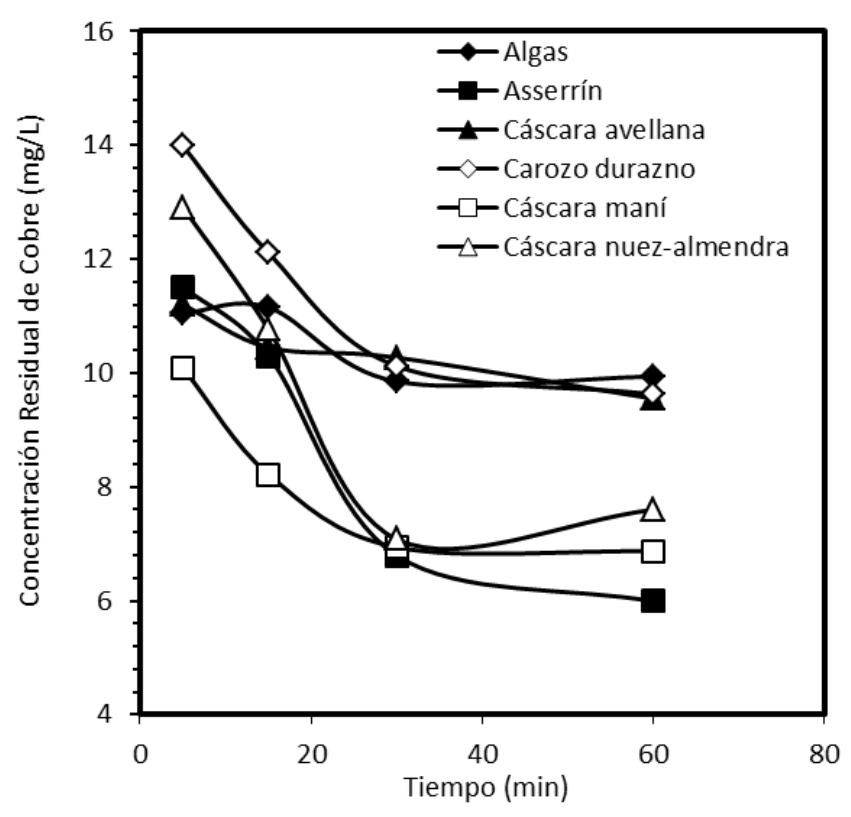

Figura 1: Efecto del tiempo de contacto en la concentración residual de cobre utilizando sorbentes orgánicos. Concentración inicial de cobre=20 mg/L; pH inicial=5,06; concentración del sorbente=20 g/L.

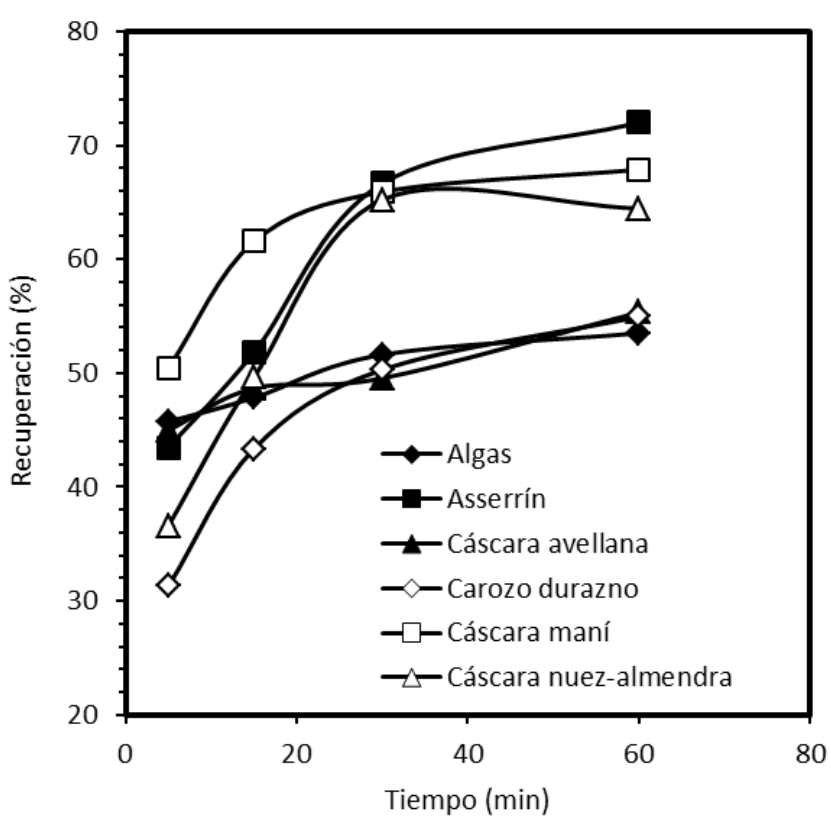

Figura 2: Efecto del tiempo de contacto en la recuperación de cobre utilizando sorbentes orgánicos. Concentración inicial de cobre $=20$ $\mathrm{mg} / \mathrm{L}$; pH inicial=5,06; concentración del sorbente $=20 \mathrm{~g} / \mathrm{L}$.

\subsection{Concentración del sorbente}

El efecto de la concentración del sorbente sobre la concentración residual de cobre se muestra en la Figura 3. Se observa que la mayor reducción se obtuvo con cáscara de nuezalmendra, aserrín y cáscara de maní, disminuyendo la concentración residual de cobre desde 20 $\mathrm{mg} / \mathrm{L}$ a valores inferiores a $8 \mathrm{mg} / \mathrm{L}$, con $40 \mathrm{~g} / \mathrm{L}$ de sorbente.

El comportamiento de todos los sorbentes a bajas concentraciones $(10 \mathrm{~g} / \mathrm{L})$ fue muy similar, obteniéndose concentraciones residuales de cobre que varió entre 12,79 y 9,71 mg/L. Para complementar los resultados de la Figura 3, en la Figura 4 se presenta la variación de la remoción de cobre en función de la concentración del sorbente. Se observa que a baja concentración de sorbente $(10 \mathrm{~g} / \mathrm{L})$ la remoción de cobre más alta obtenida fue levemente superior a $50 \%$, esto se debe a que se dispone de una menor área superficial de sorbente para captar iones de cobre, lo que se traduce en bajas remociones. El aumento más significativo en la remoción de cobre, al aumentar la concentración del sorbente, se obtubo con cáscara de nuez-almendra y aserrín. En la Figura 4 también se observa que al emplear algas y cáscara de maní no se obtuvo variación notoria en la remoción de cobre, la que alcanzó valores del orden de 52 y $59 \%$.

Debe señalarse que, de acuerdo a lo observado en la Figura 2, los mejores resultados de sorción se alcanzaron en tiempos de contacto de 30 y 60 minutos. Sin embargo, a pesar de que estos tiempos permiten representar mejor los resultados de este estudio, se eligió un tiempo de contacto de 15 minutos para realizar los ensayos de los resultados que se muestran en las Figuras 3 y 4 . Explicando lo anterior, aunque al incrementarse el tiempo de contacto para valores mayores a 15 minutos se aprecia una diferencia notoria en los resultados alcanzados con aserrín y cáscara de nuez-almendra, y algo menos con cuesco de durazno; esa diferencia no es tan apreciable al utilizarse cáscara de maní, algas y cascara de avellana; ya que con esos sorbentes se 
observa que, al aumentar el tiempo de contacto a 30 y 60 minutos, los resultados mejoran levemente.

En la literatura se presentan estudios realizados con sorbentes orgánicos, Gaballah y Kilbertus (1995) empleando concentraciones de $20 \mathrm{~g} / \mathrm{L}$ de corteza de pino escocés modificado, quienes obtuvieron niveles de remoción de cobre del orden de $87 \%$, en pH 5 y concentración de cobre de 100 ppm. Por otra parte, Al-Asheh y Duvjak (1998) al poner en contacto corteza de pino en concentración de $9,2 \mathrm{mg} / \mathrm{mL}$ con una solución de $30 \mathrm{mg} / \mathrm{L}$ de cobre en $\mathrm{pH} 4$ obtuvieron una remoción de 0,11 $\mathrm{mmol} / \mathrm{g}$.

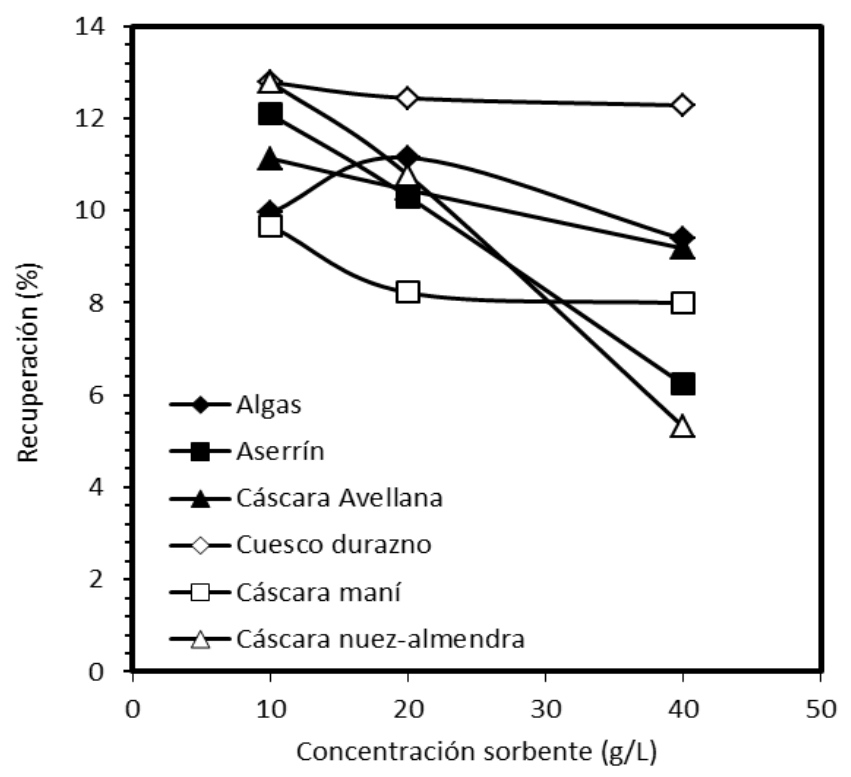

Figura 3: Efecto de la concentración de los sorbentes orgánicos en la concentración residual de cobre. Concentración inicial de cobre=20 $\mathrm{mg} / \mathrm{L}$; $\mathrm{pH}$ inicial=5,01; tiempo de contacto=15 $\mathrm{min}$.

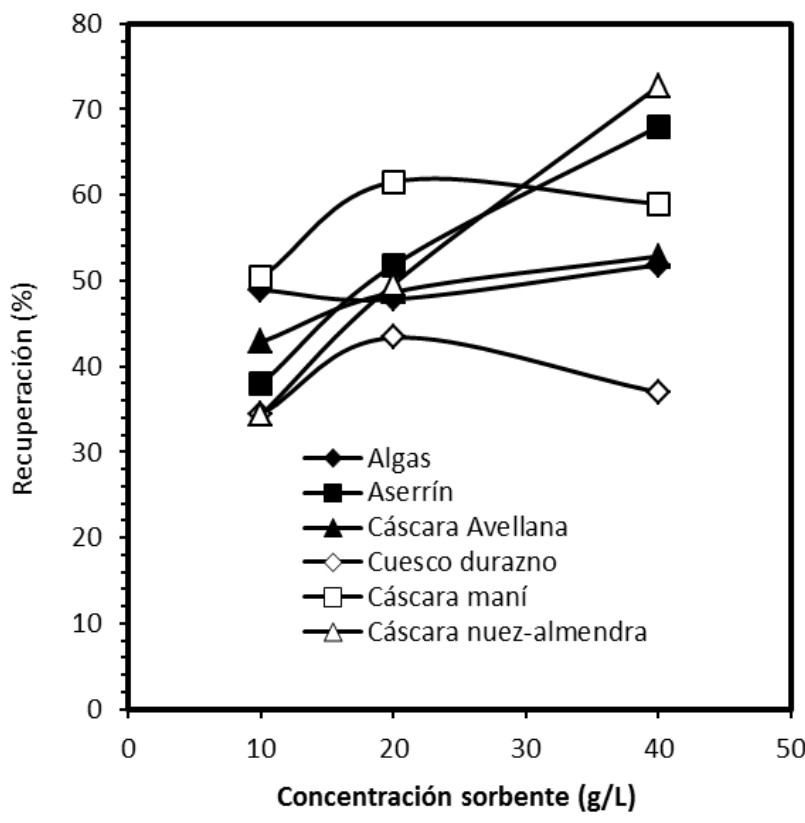

Figura 4: Efecto de la concentración de los sorbentes orgánicos en la recuperación de cobre. Concentración inicial de cobre $=20$ $\mathrm{mg} / \mathrm{L} ; \mathrm{pH}$ inicial=5,01; tiempo de contacto $=15$ $\min$.

\subsection{Efecto de la concentración inicial de cobre en la solución}

En la Figura 5 se muestra el efecto que tiene la concentración inicial de cobre de la solución sobre la captación de cobre por los diferentes sorbentes en $\mathrm{pH} 5$, tiempo de agitación de 30 minutos y concentración de sorbente de $20 \mathrm{~g} / \mathrm{L}$. En la mayoría de los sorbentes utilizados en este estudio, un tiempo de agitación de 30 minutos se considera suficiente para alcanzar el equilibrio del sistema. Se observa en la Figura 5 que al aumentar la concentración de cobre se produce un incremento en la captación de cobre. En el rango de concentración estudiado se aprecia que en general las captaciones de cobre no superan los $0,7 \mathrm{mg} / \mathrm{g}$. Los cuescos de durazno son los que presentan las menores captaciones de cobre, el resto de los sorbentes presentan en general un comportamiento bastante similar. Los valores sobre captación de cobre obtenidos en el presente estudio son inferiores a los resultados reportados en la literatura con diferentes sorbentes orgánicos, tales como residuos de manzana con y sin tratamiento (Lee y Yang, 1997), corteza de pino (Al-Sheh y Duvajak, 1998) y Rhizopus arrhizus (Sag, Kaya y Kutsal, 2000). Sin embargo, debe tenerse presente que las condiciones experimentales en que trabajaron los autores citados fueron diferentes a las del presente trabajo. 
Los datos de los equilibrios de sorción alcanzados para cada concentración inicial fueron analizados mediante las ecuaciones de las isotermas de adsorción de Langmuir, B.E.T. Y Freundlich (Tabla IV). En estas ecuaciones q representa la concentración de adsorbato adsorbido por peso de sorbente; Cf (Langmuir, BET) y Co (Freundlich) corresponden a la concentración de adsorbato en equilibrio; $\mathrm{K}$ y $1 / \mathrm{n}$ (Freundlich) constantes características del sistema; $\mathrm{K}$ (Langmuir) y $B(B E T)$ representan la capacidad máxima de adsorción en la fase sólida; b (Langmuir) y $Q$ (BET) son la constantes de energía relacionadas con el calor de adsorción. Para el ajuste de los datos experimentales se utilizó el software Statgraphics Plus 4.0. Los resultados del análisis de regresión lineal, los parámetros empíricos y los coeficientes de correlación estimados desde las ecuaciones de Langmuir, B.E.T. y Freundlich para la sorción de cobre, son presentados en la Tabla V, TablaVI y Tabla VII, respectivamente. Los coeficientes de correlación obtenidos para cada modelo fueron significativamente diferentes entre si $\left(p=4,7 \times 10^{-5}\right.$ a $\left.1,9 \times 10^{-6}\right)$. Comparando el ajuste de los datos a cada modelo y su error estadístico (ee), se obtuvo que los datos observados de $R^{2}$ se ajustaron al modelo de adsorción de Langmuir en 33.3+33,1\%, al modelo B.E.T. en $67,4 \pm 15,2 \%$ y al modelo de Freundlich en $99,1 \pm 0,8 \%$.

Según lo anterior, las ecuaciones de Langmuir y de B.E.T. no son adecuadas para explicar el fenómeno de sorción de cobre en los sistemas estudiados. Por su parte, los datos de $R^{2}$ mostraron un buen ajuste a la ecuación de Freundlich de los sorbentes utilizados en este estudio. De acuerdo a los valores de K y $\mathrm{n}$ mostrados en la Tabla VII, la constante $\mathrm{K}$, la cual da una medida de la capacidad de adsorción del sorbente, presenta un valor superior para las cáscaras de nuezalmendra $(0,032)$, resultando bastante similar para los otros materiales usados, excepto para los cuescos de duraznos que presentan un valor de $\mathrm{K}$ bastante inferior $(0,009)$. De igual forma, el valor de la constante $\mathrm{n}$ que refleja la intensidad de la adsorción, es menor para los cuescos de durazno. Aunque el modelo de Freundlich representó bien los datos de equilibrio de los sistemas estudiados ( $R^{2}$ del orden de 0,99 ), los valores de $\mathrm{n}$ obtenidos para los diferentes sorbentes fueron menores que 1 (no están en el rango recomendado $1<n<10$ ), lo que indicaría que las isotermas de Freundlich no son favorables para la sorción de los iones cobre en los sorbentes.

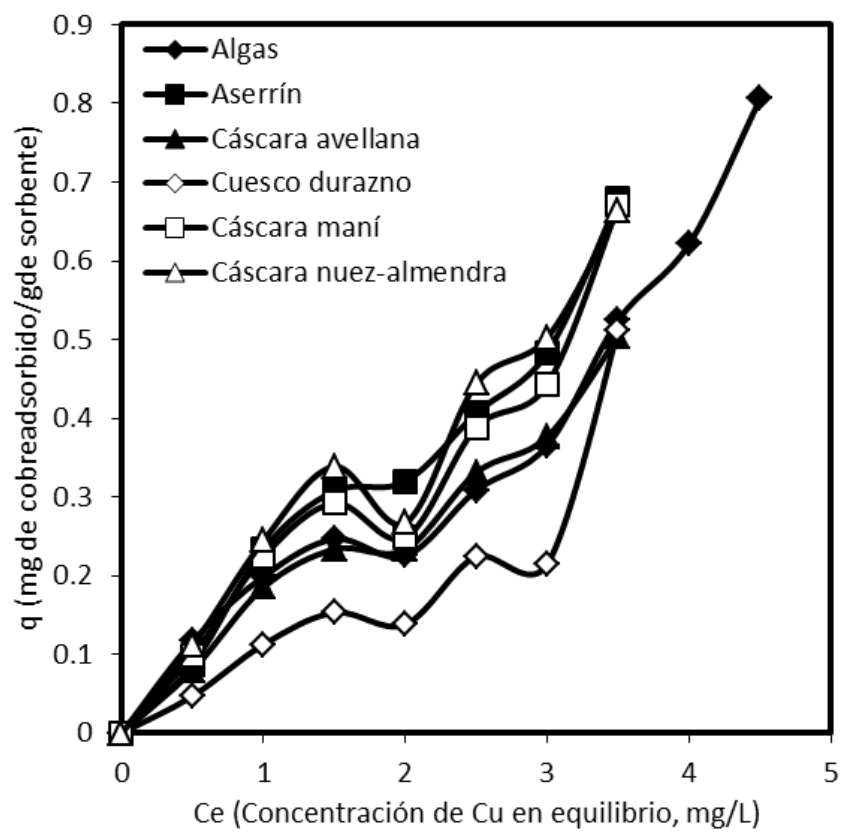

Figura 5: Efecto concentración inicial de cobre en el efluente sobre la captación de cobre, utilizando sorbentes orgánicos. pH solución $\cong 5,0$; concentración del sorbente= $20 \mathrm{~g} / \mathrm{L}$; tiempo de contacto=30 $\mathrm{min}$. 
Tabla IV: Ecuaciones de las isotermas de adsorción de Langmuir, B.E.T. y Freundlich.

\begin{tabular}{c|c|c}
\hline Modelo & Ecuación & Regresión \\
\hline Langmuir & $q=\frac{K b C_{f}}{1+b C_{f}}$ & $C_{f} / q$ versus $C_{f}$ \\
\hline BET & $q=\frac{B Q C_{f}}{\left(C_{o}-C_{f}\right)\left[1+(B-1)\left(C_{f} / C_{o}\right)\right]}$ & $C_{/} /\left(q\left(C_{o}-C_{f}\right)\right)$ versus $C_{f} / C_{o}$ \\
\hline Freundlich & $q=K C_{o}{ }^{1 / n}$ & $\log q$ versus Log Co \\
\hline
\end{tabular}

Tabla V: Coeficiente de correlación y datos empíricos estimados desde la ecuación de Langmuir, para la sorción de cobre.

\begin{tabular}{l|c|c|c}
\hline \multirow{2}{*}{ Sorbente } & \multicolumn{2}{|c|}{ PARÁMETROS } & \multirow{2}{*}{$\begin{array}{c}\text { Coeficiente de } \\
\text { correlación }\end{array}$} \\
\cline { 2 - 3 } & $B$ & $K$ & $-0,468$ \\
\hline Algas & $-0,016$ & $-2,739$ & $-0,652$ \\
Aserrín & 0,000 & $-0,767$ & $-0,332$ \\
Cáscara avellana & 0.000 & -3.946 & $-0,591$ \\
Cuesco durazno & -0.042 & -0.399 & $-0,216$ \\
Cáscara maní & -0.025 & -2.714 & 0,258 \\
Cáscara nuez-almendra & 0.051 & 2.240 & \\
\hline
\end{tabular}

Tabla VI: Coeficiente de correlación y datos empíricos estimados desde la ecuación de B.E.T., para la sorción de cobre.

\begin{tabular}{l|c|c|c}
\hline \multirow{2}{*}{ Sorbente } & \multicolumn{2}{|c|}{ PARÁMETROS } & Coeficiente de \\
\cline { 2 - 3 } & $Q$ & $B$ & correlación \\
\hline Algas & 0,026 & $-1,270$ & 0,800 \\
Aserrín & 0,025 & $-2,172$ & 0,952 \\
Cáscaras avellana & 0,019 & $-1,245$ & 0,593 \\
Cuesco durazno & 0,016 & $-0,916$ & 0,599 \\
Cáscara maní & 0,056 & $-2,553$ & 0,580 \\
Cáscara nuez-almendra & 0,100 & $-4,765$ & 0,548 \\
\hline
\end{tabular}

Tabla VII: Coeficiente de correlación y datos empíricos estimados desde la ecuación de Freundlich, para la sorción de cobre.

\begin{tabular}{l|c|c|c}
\hline \multirow{2}{*}{ Sorbente } & \multicolumn{2}{|c|}{ PARÁMETROS } & \multirow{2}{*}{$\begin{array}{c}\text { Coeficiente de } \\
\text { correlación }\end{array}$} \\
\cline { 2 - 3 } & $K$ & $n$ & 0,995 \\
\hline Algas & 0,021 & 0,931 & 0,998 \\
Aserrín & 0,023 & 0,870 & 0,997 \\
Cáscara avellana & 0,023 & 0,957 & 0,978 \\
Cuesco durazno & 0,009 & 0,797 & 0,991 \\
Cáscara maní & 0,026 & 0,943 & 0,984 \\
Cáscara nuez-almendra & 0,032 & 0,984 & \\
\hline
\end{tabular}




\subsection{Efecto de la fuerza iónica}

La influencia de la fuerza iónica de la solución en la remoción de cobre se estudió mediante la adición de diferentes concentraciones de $\mathrm{NaCl}$. En la Figura 6 se muestra el efecto de la fuerza iónica sobre la remoción de cobre en la ausencia y presencia de 0,25 y $12,8 \mathrm{~g} / \mathrm{L}$ de sodio, con soluciones de cobre de $20 \mathrm{mg} / \mathrm{L}$, concentración de sorbente de $20 \mathrm{~g} / \mathrm{L}$, pH 5,2 y tiempo de agitación de 15 minutos. Se observa que al emplear aserrín una baja concentración de cloruro de sodio prácticamente no afecta la remoción de cobre, sin embargo, al aumentar la fuerza iónica la remoción disminuye notoriamente. Esto puede atribuirse al efecto competitivo que tendría los iones sodio con respecto a los iones cobre por ligarse al aserrín. La presencia de $\mathrm{NaCl}$ en concentraciones de $0,01 \mathrm{~N}$ (0,24 g/L de sodio) afecta la eliminación de cobre al emplear cáscaras de maní, apreciándose claramente que la remoción del metal disminuye al aumentar la fuerza iónica de la solución. Por esta razón, debido a que la adsorción de cobre en el sorbente se muestra sensible al cambio de la fuerza iónica, posiblemente uno de los mecanismos importantes involucrados en la remoción de cobre con este sorbente sea la atracción electrostática. En el caso de las algas, la Figura 6 muestra que al utilizar $0,25 \mathrm{~g} / \mathrm{L}$ de sodio la remoción de cobre baja en forma notoria a valores inferiores a 50\%, sin embargo, al aumentar la fuerza iónica de la solución (12,8 g/L de sodio), la remoción prácticamente no continua disminuyendo. En la literatura, Lee y Yang (1997) reportaron el efecto de la fuerza iónica en la remoción de cobre utilizando residuos de manzana modificados, señalando que con concentraciones de $\mathrm{NaCl}$ inferiores a $0,1 \mathrm{~N}$ no se producía un decrecimiento significativo en la remoción de cobre, sin embargo un fuerte aumento de la fuerza iónica sobre $0,1 \mathrm{~N}$ de $\mathrm{NaCl}$ disminuía en alrededor de un $40 \%$ la capacidad de remoción de cobre de los residuos de manzana. Estos autores indicaron que si el mecanismo significativo en la remoción del metal es la atracción electrostática, la adsorción es sensible a los cambios de concentración del $\mathrm{NaCl}$.

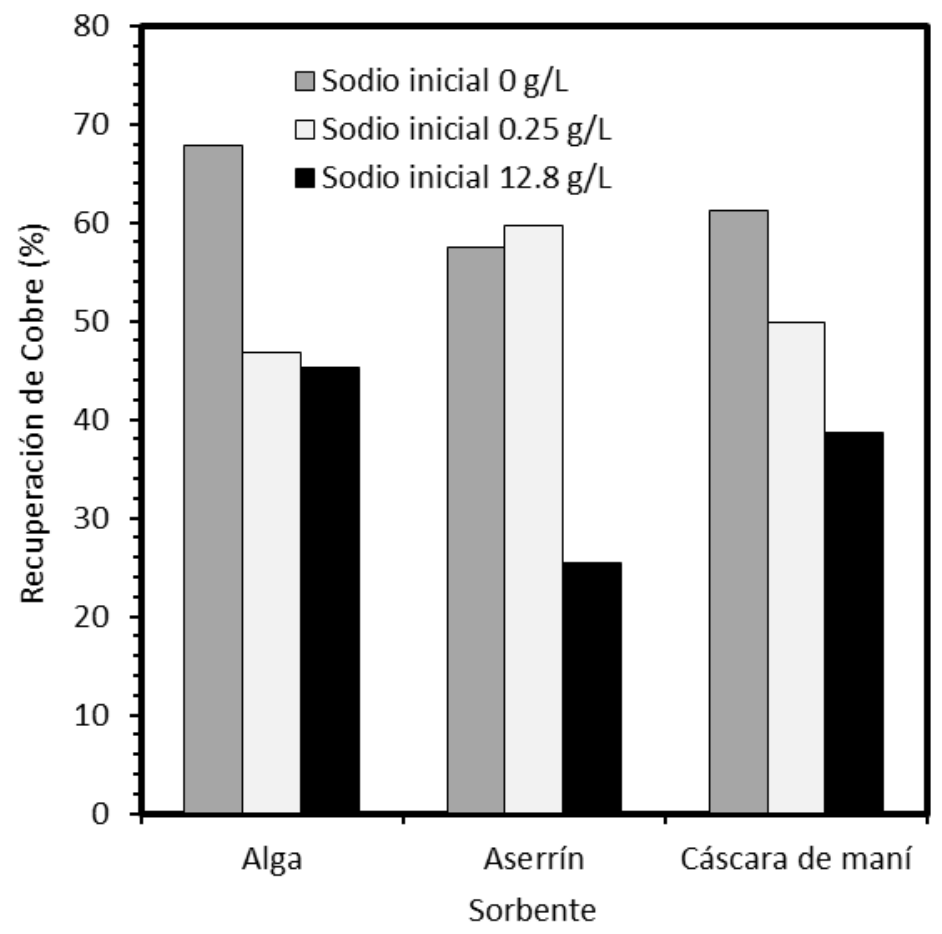

Figura 6: Efecto de la fuerza iónica en la remoción de cobre. Concentración inicial de cobre=20 $\mathrm{mg} / \mathrm{L}$,

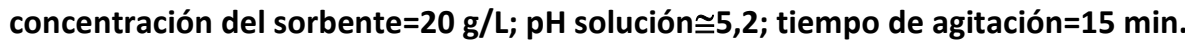




\section{CONCLUSIONES}

Los sorbentes orgánicos utilizados en este estudio permiten la remoción parcial de los iones de cobre desde las soluciones preparadas artificialmente. En general, los mejores resultados fueron obtenidos con aserrín, cáscara de maní y cáscara de nuez-almendra, mientras que, la menor remoción de cobre se alcanzó con cuesco de durazno.

Remociones de cobre del orden de 30 a 50\% se obtuvieron para un tiempo de agitación de 5 minutos, mientras que al incrementar el tiempo a 60 minutos las remociones de cobre aumentaron a valores comprendidos entre 50 y $70 \%$.

Al aumentar la concentración del sorbente desde $20 \mathrm{mg} / \mathrm{L}$ a $40 \mathrm{mg} / \mathrm{L}$ las remociones de cobre con cáscara de avellana, algas, cuesco de durazno y cáscara de maní prácticamente no se ven afectadas, en cambio los resultados alcanzados con las cáscara de nuez-almendra y aserrín mejoran notoriamente al incrementar la concentración del sorbente.

Los datos de equilibrios de sorción obtenidos para cada concentración inicial de cobre se analizaron mediante las ecuaciones de isotermas de adsorción de Langmuir, B.E.T. y Freundlich. Las ecuaciones de Langmuir y B.E.T. no fueron adecuadas para representar la sorción de cobre en los sistemas estudiados, en cambio la aplicación de la ecuación de Freundlich originó coeficientes de correlación $\left(R^{2}\right)$ del orden de 0,99 , por lo tanto el proceso de sorción de cobre con los sorbentes orgánicos estudiados se representa en forma adecuada por el modelo de Freundlich.

En cuanto al efecto de la fuerza iónica de la solución, se encontró que la presencia de iones sodio en la solución afecta la remoción de cobre que disminuye con el aumento de la fuerza iónica.

\section{REFERENCIAS}

Al-Asheh, S., y Duvjak, Z. (1998). Binary metal sorption by pine bark: study of equilibria and mechanism. Separation Science and Technology, 33(9),1303-1329.

Alguacil, J.F., y Merino, Y. (1998). Biotratamiento de contaminantes de origen orgánico. Revista de Metalurgia, 34(5), 428-436.

Bal, Y., Bal, K.E., Laarbi-Bouamrane, O., y Lallam, A. (2006). Copper (II) uptake by Pleurotas mutilus biomasa, chitin and chitosan. Minerals Engineering, 19(14), 1456-1458.

Bandela, N.N., Bagrekar, M.G., Jogdand, O.K., y Kaushik, G. (2016). Removal of copper from aqueous solution using local agricultural wastes as low cost adsorbent. Journal of Materials Enviromental and Science, 7(6), 1972-1978.

Berder, J., y Phillips, P. (1996). Biotratamiento de desagues de mina. Mining, Abril, 31-33.

Castellan, G. (1975). Fisicoquímica. Puerto Rico: Fondo Educativo Interamericano, S. A.

Candan, M., Tay, F., Avan, I., y Tay, T. (2017). Removal of copper(II) and nickel(II) ions from aqueous solution using non-living lichen Ramalina fraxinea biomass: investigation of kinetics and sorption isotherms. Desalination and Water Treatment, 75, 148-157.

Deng, L.P., Su, Y.Y., Su, H., Wang, X.T., y Zhu, X.B. (2006). Biosorption of copper (II) and lead (II) from aqueous solutions by nonliving green algae Cladophora fascicularis: Equilibrium, kinetics and environmental effects. Adsorption-Journal of the International Adsorption Society, 12(4), 267-277.

Doménech, X. (1995). Química del suelo. Madrid, España: Ediciones Miraguano.

Estefandian, H., Javadian, H., Parvini, M., Khoshandam, B., y Katal, R. (2013). Batch and column removal of copper by modified brown algae sargassum bevanom from aqueous solution. Asia-Pacific Journal of Chemical Engineering, 8(5), 665-678. 
Fagundes-Klen, M.R., Veit, M.T., Borba, C.E., Bergamasco, R., Vaz, L.G.D., y da Silva, E.A. (2010). Copper biosorption by biomass of marine alga: Study of equilibrium and kinetics in batch system and adsorption/desorption cycles in fixed bed column. Water Air and Soil Pollution, 213(1-4), 15-26.

Gabaldón, C., Marzal, P., y Seco, A. (2000). Cadmium and cooper removal by a granular activated carbon in laboratory column systems. Separation Science and Technology, 35(7), 1039-1053.

Gaballah, L., y Kilbertus, G. (1995). Elimination of As, $\mathrm{Hg}$ and $\mathrm{Zn}$ from synthetic solutions and industrial efluents using modified bark. Conference: The Mineral, Metals \& Materials Society (TMS), Las Vegas, United States, 12-16 Feb, 15-26.

Ho, Y., Chiang, Ch., y Hsu, Y. (2001). Sorption kinetics for dye removal from aqueous solution using activated clay. Separation Science and Technology, 36(11), 2473-2488.

Ho, Y., Ng, J., y Mckay. (2001). Removal of lead (II) from effluents by sorption on peat using second-order kinetics. Separation Science and Technology, 36(2), 241-261.

Jolly, G.; Dupont, L. Aplincourt, M., y Lambert, J. (2006). Improved Cu and Zn sorption on oxidized wheat lignocellulose. Environmental Chemistry Letters, 4(4), 219-223.

Kamar, F.H., y Nechifor, A.C. (2015). Removal of copper ions from industrial wastewater using walnut shells as a natural adsorbent material. UPB Scientific Bulletin, Series B, 77(3), 142-150.

Khan, A.M., Ahmad, C. S., Farooq, U., Mahmood, K., Sarfraze, M., Balkhair, K.S., y Ashraf, M.A. (2015). Removal of metallic elements from industrial waste water through biomass and clay. Frontier in Life Science, 8(3), 223-230.

Kondo, K., Sumi, H., y Matsumoto, M. (1996). Adsorption characteristics of metal ions on chitosan chemically modified by D-galactose. Separation Science and Technology, 31(12), 1771-1775.

Kushwah, A., y Srivastav, J.K. Biosorption of copper ions by green algae Spirogyra. (2015). International Journal of Chemical Studies, 3(3), 36-38.

Lahari, S.B., King, P., y Prasad, V.S.R.K. (2011). Biosorption of copper from aqueous solution by chaetomorpha antennina algae biomass. Iranian Journal of Environmental Helath Science \& Engineering, 8(4), 383-392.

Lee, S., y Yang, J. (1997). Removal of copper in aqueous solution by apple wastes. Separation Science and Technology, 32(8), 1371-1387.

Luna, A.; da Costa, A.C.A.; Henriques, C.A., y Herbst, M.H. (2007). Electrón paramagnetic resonante and atomic absorption spectrometry as tools for the investigation of $\mathrm{Cu}$ (II) biosorption by Sargassum filipendula. Hidrometallurgy, 86(1-2), 105-113.

Mohammed, T.J., Azeez, R.A., y Lutffe, T. (2015). Biosorption of copper from synthesized wastewater using agricultura waste (roasted date pits). International Journal of Recent Scientific Research, 6(3), 3063-3068.

Radu, N., Bondar, E., Meghea, A., y Rau, I. (2006). Removal of copper and cobalt ions from aqueous solutions by biosorption. Revista de Chimie, 57(7), 711-717.

Sag, Y. Kaya, A., y Kutsal, T. (2000). Biosorption of lead (II), nickel (II), and copper (II) on Rhizopus Arrhizus from binary and ternary metal mixtures. Separation Science and Technology, 35(16), 2601-2617.

Sag, Y., Atacoglu, I., y Kutsal, T. (1999). Simultaneous Biosorption of chromium (VI) and copper (II) on Rhizopus Arrhizus in packed column reactor: Application of the competitive Freundlich model. Separation Science and Technology, 34(16), 3155-3171.

Saeed, A., Akhter, M.W., y lqbal, M. (2005). Removal and recovery of heavy metals from aqueous solution using papaya wood as a new biosorbent. Separation and Purification Technology, 45(1), 25-31. 
Sheng, P.X., Wee, K.H., Ting, Y.P., y Chen, J.P. (2008). Biosorption of copper by immobilized marine algal biomass. Chemical Engineering Journal, 136(2-3), 156-163.

Singh, J., Ali, A., y Kumar, R. (2013). Removal of $\mathrm{Ni}^{2+}, \mathrm{Cu}^{2+}$ and $\mathrm{Zn}^{2+}$ using different agricultural residues: kinetics, isotherm modeling and mechanism via chemical blocking. Asian Journal of Chemistry, 25(11), 6377-6386.

Singha, B., y Das, S.K. (2013). Adsorptive removal of $\mathrm{Cu}(\mathrm{II})$ from aqueous solution and industrial effluent using natural/agricultural wastes. Colloids and Surfaces B-Biointerfaces, 107, 97-106.

Tapia, P, Santander, M., Pavez, O., Guzmán, D., y Romero, L. (2011). Biosorción de iones cobre con biomasa de algas y orujos deshidratados. Revista de Metalurgia, 47(1), 15-28.

Tripathi, A., y Ranjan, M.R. (2015). Heavy metal removal from wastewater using low cost adsorbents. Journal of Bioremediation and Biodegradation, 6(6) 315-319.

Vilar, V.J.P., Botelho, C.M.S., Pinheiro, J.P.S., Domingos, R.F., y Boaventura, R.A.R. (2009). Copper removal by algal biomass: biosorbents characterization and equilibrium modeling. Journal of Hazardous Materials, 163(2), 1113-1122.

Zhu, C.S., Wang, L.P., y Chen, W.B. (2009). Removal of $\mathrm{Cu}(\mathrm{II})$ from aqueous solution by agricultural by-product: peanut hull. Journal of Hazardous Materials, 168(2-3), 739-746. 